\title{
The Multiple Steiner TSP with order constraints: complexity and optimization algorithms
}

\author{
Virginie Gabrel ${ }^{1}$ - A. Ridha Mahjoub ${ }^{1} \cdot$ Raouia Taktak $^{2}$ (D) $\cdot$ Eduardo Uchoa $^{3}$
}

๑) Springer-Verlag GmbH Germany, part of Springer Nature 2020

\begin{abstract}
We consider a variant of the Travelling Salesman Problem (TSP), the Multiple Steiner TSP with Order constraints (MSTSPO). Consider a weighted undirected graph and a set of salesmen, and each salesman is associated with a set of compulsory vertices to visit, called terminals. The MSTSPO consists in finding a minimum-cost subgraph containing for each salesman a tour going in a specified order through its terminals. Along with its importance from a theoretical point of view, the problem is also challenging in practice since it has applications in telecommunication networks. We show that the problem is NP-hard even for a single salesman and propose integer programming formulations. We then devise both Branch-and-Cut and Branch-and-Price algorithms to solve the problem. The extensive computational results are presented, showing the efficiency of our algorithms.
\end{abstract}

Keywords Steiner TSP · Order constraints · Integer linear programming · Branch-and-Price algorithm · Branch-and-Cut algorithm

\section{Introduction and related work}

\subsection{Related TSP variants}

Given a weighted undirected graph $G=(V, E)$, the Traveling Salesman Problem, or TSP for short, consists in finding a minimum-cost hamiltonian circuit or tour. The TSP has been one of the most intensively studied optimization problems, and several books have been written on it (Applegate et al. 2011; Gutin and Punnen 2006). Through the years, the

Communicated by V. Loia.

Raouia Taktak

raouia.taktak@isims.usf.tn

Virginie Gabrel

virginie.gabrel@lamsade.dauphine.fr

A. Ridha Mahjoub

ridha.mahjoub@lamsade.dauphine.fr

Eduardo Uchoa

eduardo.uchoa@gmail.com

1 PSL Research University, CNRS, LAMSADE, Université Paris-Dauphine, 75016 Paris, France

2 ISIMS and LT2S/CRNS, Université de Sfax, Sfax, Tunisia

3 Departamento de Engenharia de Produção, Universidade Federal Fluminense, Niterói, RJ, Brasil
TSP has been efficiently solved mainly using Branch-andCut algorithms such as described in Grötschel and Padberg (1979a,b), Naddef (2007) and Padberg and Rinaldi (1991). The majority of these works are based on the natural formulation proposed by Dantzig et al. (1954), a formulation using an exponential number of constraints that necessitates a cutting-plane phase. In the literature, we also find a variety of compact formulations for the TSP that are formulations using a polynomial number of variables and constraints, see, for example, Gouveia and Voß (1995), Öncan et al. (2009) and Orman and Williams (2006). Moreover, due to its several applications in various fields, many variants of the problem have been studied.

One of these variants is the Steiner Traveling Salesman Problem (STSP) in which only a given subset $T \subset V$ of nodes, called terminals, must be visited in a minimum-weight closed walk, eventually visiting some nodes and edges more than once. The STSP was first introduced by Cornuéjols et al. (1985) who study the problem in the graphical case and investigate its polyhedron in series-parallel graphs. Later, Mahjoub and Baiou give a complete polyhedral description of the STSP in series-parallel graphs (Baïou and Mahjoub 2002). Salazar-González (2003) investigates some polyhedral results and devises a lifting method to construct facets from known ones for the TSP. Steinová (2012) proposes some approximation results for the problem. Letchford et al. (2013) 
introduce compact formulations for the STSP obtained from the ones known for the TSP. Recently, Interian and Ribeiro (2017) propose efficient GRASP heuristic-based algorithm for the problem.

Another important variant of the TSP is the TSP with precedence constraints. This arises when the circuit starts and ends at a given node, and precedence constraints between some pairs of nodes are considered. This variant was also widely studied in the literature. Balas et al. (1995) study the polytope of the Asymmetric TSP with precedence constraints. Ascheuer et al. (2000) propose a Branch-and-Cut algorithm to solve the problem. Moon et al. (2002) propose an efficient genetic algorithm to solve the problem heuristically. Gouveia and Pesneau (2006) give extended formulations for the Asymmetric TSP with precedence constraints and describe a Branch-and-Cut algorithm for the problem. In a further work, Gouveia et al. (2018) propose new formulations for the Asymmetric TSP and the related precedence constrained version combining precedence variable-based formulations with network flow based formulations.

A further interesting variant is the so-called Multiple TSP which consists in finding a set of tours for a predefined number of salesmen, each tour starting from and coming back to a depot node while visiting exactly once the other intermediate nodes. Bektas (2006) presents a survey of formulations and solution approaches for the problem, assuming that all salesmen start from the same depot. Benavent and Martínez (2013) study the problem when salesmen are assumed to start from different depots. The authors propose an ILP formulation for the problem. Several families of valid inequalities are identified, and a substantial polyhedral study is presented. The authors devise an efficient Branch-and-Cut algorithm to solve the problem. Sarin et al. (2014) study the multiple asymmetric TSP with precedence constraints and investigate the performances of 32 formulations modeling the problem.

\subsection{Problem statement and practical motivation}

In this paper, we study the Multiple Steiner TSP with Order constraints (MSTSPO), closely related to the previously mentioned variants of the TSP. The problem was introduced in Borne et al. (2011, 2013), motivated by reliability issues in multilayer telecommunication networks. Mahjoub et al. (2019) propose an ILP compact formulation for the MSTSPO. The formulation is based on a layered view of the problem. An extensive computational study shows the efficiency of the proposed formulation in solving hard instances.

\subsubsection{Practical motivation}

The problem takes origin from a reoptimization phase in an intern tool for network optimization developed in Orange Labs, France. Consider a two-layer IP-over-optical network.
The optical network is composed of optical switches, also called optical cross-connects (OXC), interconnected by optical links. These optical switches are connected to the IP routers through logical control interfaces called user-network interfaces (UNI). The optical network essentially provides point-to-point connectivity between routers in the form of fixed bandwidth lightpaths. These lightpaths define the topology of the IP network. Each router in the IP network is associated with an optical switch, and to each link between two routers corresponds to a path between the corresponding switches in the optical network.

Figure 1 shows an example of an IP-over-optical network. The IP layer (also called logical or virtual layer) contains four routers denoted by $R_{1}, R_{2}, R_{3}$ and $R_{4}$. The optical layer (also called physical layer) holds seven optical switches denoted $S_{1}$ to $S_{7}$. Optical switches $S_{1}, S_{2}, S_{3}, S_{4}$ correspond to the routers $R_{1}, R_{2}, R_{3}, R_{4}$, respectively. Optical switches communicate with IP routers through the UNI interfaces. $\left(R_{1}, R_{2}\right),\left(R_{2}, R_{3}\right)$ and $\left(R_{1}, R_{3}\right)$ represent virtual links in the logical layer. These links are ensured by lightpaths in the optical layer; $\left(R_{1}, R_{2}\right)$ is physically routed by $\left(S_{1}, S_{2}\right)$, $\left(R_{2}, R_{3}\right)$ by $\left(S_{2}, S_{7}, S_{3}\right)$ and $\left(R_{1}, R_{3}\right)$ by $\left(S_{1}, S_{5}, S_{7}, S_{3}\right)$. Note that the network is not survivable. In fact, if node $S_{7}$ in the optical layer breaks down, the physical paths from $S_{1}$ to $S_{3}$ and from $S_{2}$ to $S_{3}$ are interrupted. Consequently, the virtual links $\left(R_{1}, R_{3}\right)$ and $\left(R_{2}, R_{3}\right)$ in the logical layer will break down as well. In consequence, survivability strategies have to be considered, mainly through providing two-node disjoint paths during the routing process.

\subsubsection{Problem statement}

Consider an IP-over-Optical network and a set of demands in the IP layer. Assume that for each demand, we know twonode disjoint paths routing it in the IP layer. The problem consists in finding, for every demand, two-node disjoint paths routing it in the optical layer. These paths must go through the optical switches corresponding to the routers visited in the IP-layer and respect the order of these routers.

Now by considering each demand as a salesman, and each cycle formed by the two disjoint paths respecting the required order of the switches, one has to determine in the optical layer, as a tour for the salesman, this telecommunication problem is nothing but the MSTSPO.

Let $G=(V, E)$ be an undirected weighted graph, and $K$ a set of salesmen. With each salesman $k \in K$, it is associated with a set $T_{k} \subseteq V$ of terminals. The vertices in $S_{k}=V \backslash T_{k}$ are called Steiner vertices. The MSTSPO consists in finding a set of edges $F \subseteq E$, with minimum total weight, such that for each salesman $k \in K$ there is a tour that visits all terminals in $T_{k}$ in a predefined cyclic order. Steiner vertices not belonging to $T_{k}$ are optional. They may be traversed between visits and terminals. Moreover, as in the classical STSP, and due to 
Fig. 1 Example of multilayer IP-over-optical network

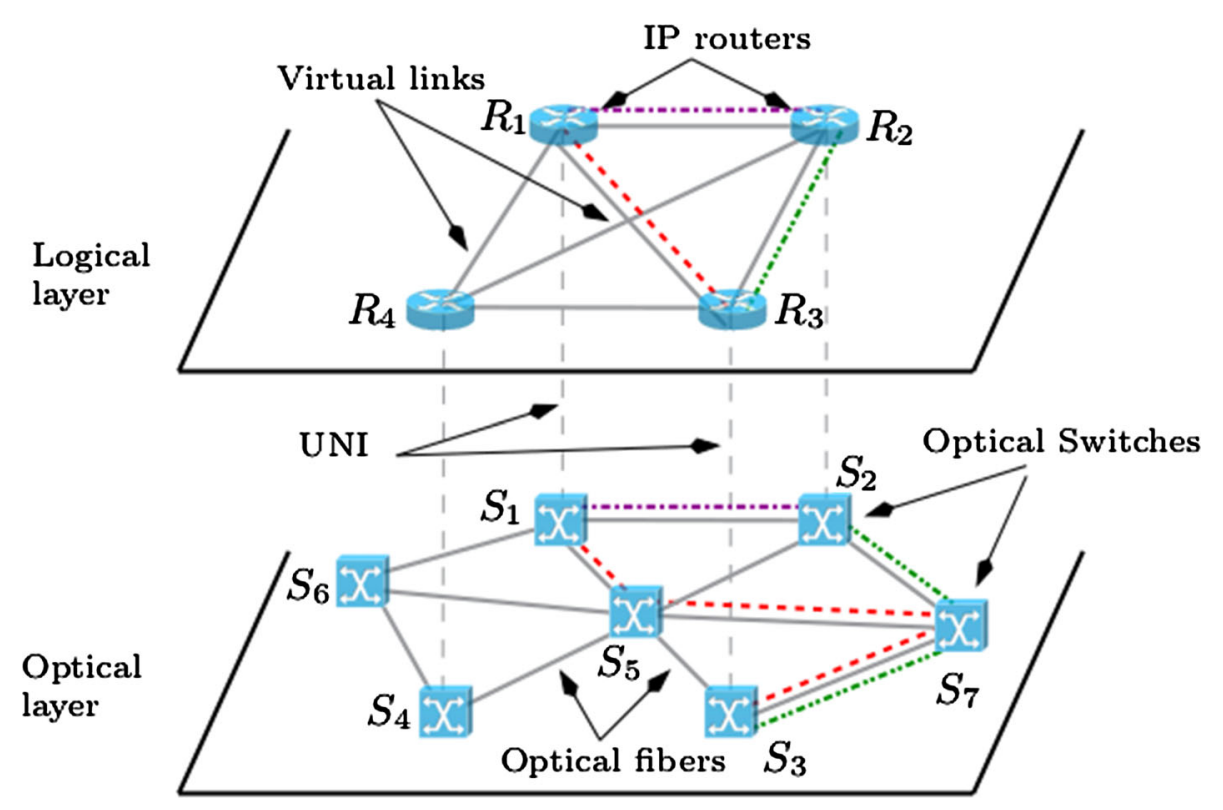

some survivability restrictions, tours must be elementary, that is, vertices and edges are not allowed to be visited more than once. In the sequel, STSPO will simply denote the Steiner TSP with Order constraints, that is, when $|K|=1$ (i.e., only one salesman). Even the STSPO is proved to be NP-hard by a reduction from the $k$-vertex disjoint paths problem .

\subsection{Paper organization}

This paper is organized as follows. In Sect. 2, we state the problem, give the notations that will be used along the paper and determine its complexity. In Sect. 3, we give two integer programming formulations. The first one, called cut formulation, is given in terms of routing variables and gives rise to Branch-and-Cut algorithm. The second is a path formulation and gives rise to a Branch-and-Price algorithm discussed in Sect. 4. In Sect. 4, we also discuss the pricing problem and prove that it reduces to a shortest path problem that can be solved in polynomial time. We also devise a branching scheme that preserves the tractability of the pricing problem and describe a primal heuristic. A computational study comparing both Branch-and-Cut and Branch-and-Price algorithms is presented and discussed in Sect. 5. The paper is concluded by some remarks given in Sect. 6 .

\section{The MSTSPO}

\subsection{Problem definition}

Let $G=(V, E)$ be an undirected graph where each edge $e \in E$ has a nonnegative cost $c(e)$. We assume given a set $K$ of salesmen, each having to visit a set of terminals
$T_{k}=\left\{v_{1}^{k}, v_{2}^{k}, \ldots, v_{\left|T_{k}\right|}^{k}\right\} \subseteq V, k \in K$. The order of terminal visitation for each tour (salesman) is assumed to follow the cyclic order of the indices $j$ in $v_{j}^{k}$. The Multiple Steiner TSP with Order constraints (MSTSPO) consists in finding a minimum-cost subgraph in $G$ such that for each salesman $k \in K$, a tour passing in order through all its terminals is calculated. The tours must be elementary, and no edge or node can be visited more than once.

In order to illustrate the problem, Fig. 2 a represents a graph $G$ composed of ten vertices $V=\left\{v_{1}, v_{2}, \ldots, v_{10}\right\}$. Edge costs are indicated. There are $|K|=3$ salesmen associated, respectively, with the following ordered terminals $T_{1}=\left\{v_{1}, v_{8}, v_{10}, v_{4}\right\}, T_{2}=\left\{v_{1}, v_{3}, v_{2}, v_{6}\right\}$, and $T_{3}=$ $\left\{v_{2}, v_{5}, v_{7}\right\}$. Figure $2 \mathrm{~b}$ depicts the optimal solution for this instance, with an objective function equal to 11 . The salesmen's tours contained in that subgraph are the following:

- Salesman 1: $\left\{\mathbf{v}_{\mathbf{1}}, v_{2}, \mathbf{v}_{\mathbf{8}}, v_{7}, \mathbf{v}_{\mathbf{1 0}}, \mathbf{v}_{\mathbf{4}}, v_{6}, \mathbf{v}_{\mathbf{1}}\right\}$,

- Salesman 2: $\left\{\mathbf{v}_{\mathbf{1}}, v_{5}, \mathbf{v}_{\mathbf{3}}, v_{10}, v_{7}, v_{8}, \mathbf{v}_{\mathbf{2}}, v_{4}, \mathbf{v}_{\mathbf{6}}, \mathbf{v}_{\mathbf{1}}\right\}$,

- Salesman 3: $\left\{\mathbf{v}_{\mathbf{2}}, v_{1}, \mathbf{v}_{\mathbf{5}}, v_{3}, v_{10}, \mathbf{v}_{\mathbf{7}}, v_{8}, \mathbf{v}_{\mathbf{2}}\right\}$,

where terminals for each salesman are in bold and the other vertices are Steiner (or optional). Note that for each salesman, all the terminals are visited in the predefined order, and that the resulting tour is elementary.

\subsection{Further notations}

If $k \in K$ and $j \in\left\{1,2, \ldots,\left|T_{k}\right|\right\}$, we call $q_{j}^{k}=\left(v_{j}^{k}, v_{j+1}^{k}\right)$ a section given by the two successive terminals $v_{j}^{k}$ and $v_{j+1}^{k}$, where $v_{\left|T_{k}\right|+1}^{k}$ refers to $v_{1}^{k}$. Each tour of a salesman $k \in K$ can be seen as union of vertex disjoint paths between nodes of sections. Let $\mathcal{T}_{k}$ be the set of all the sections of tour $k \in$ 
Fig. 2 Illustration of a MSTSPO instance and its optimal solution
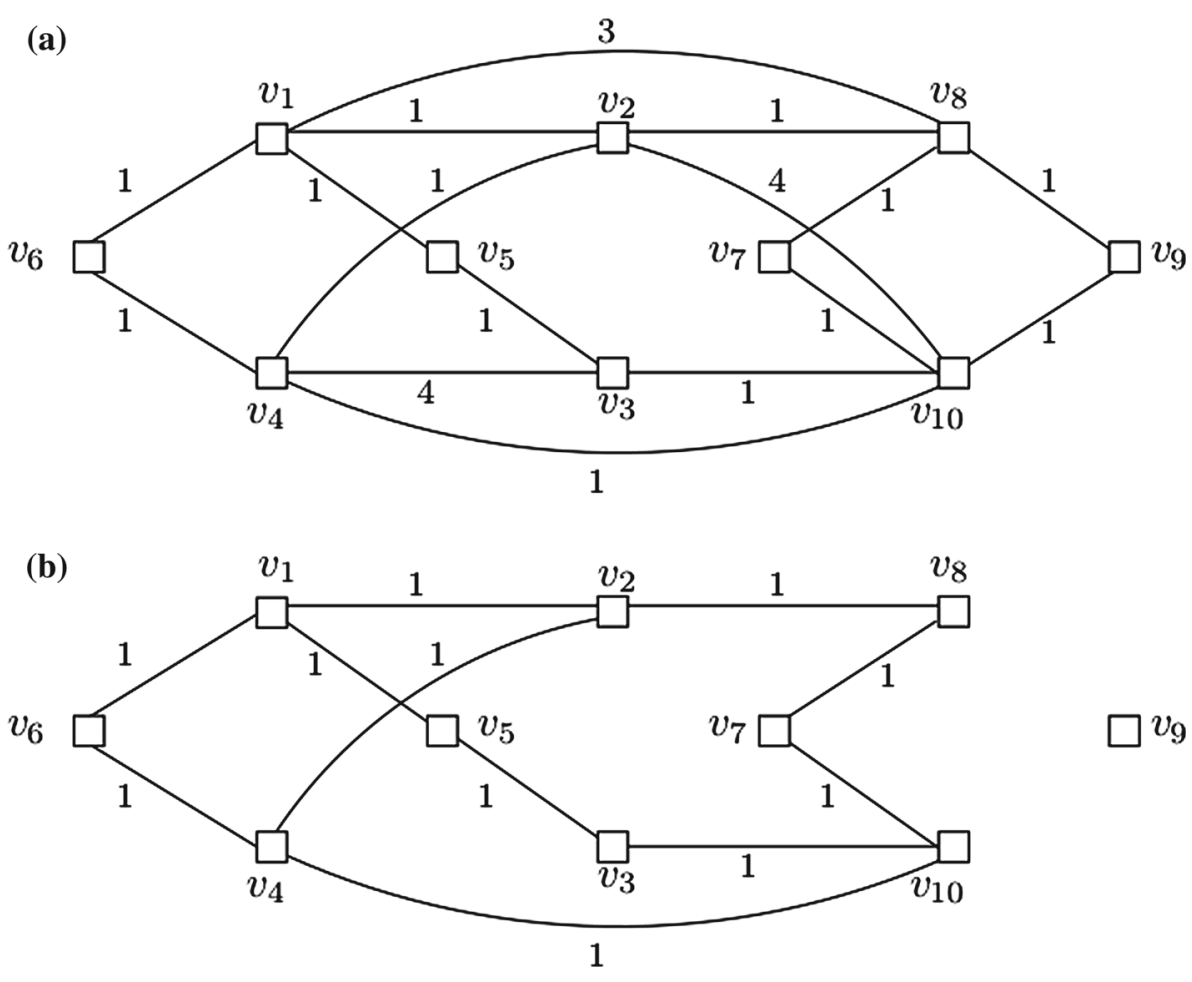

$K$. In order to simplify, in the sequel we simply note $q=$ $(u, v) \in \mathcal{T}_{k}$ to refer to a section of $k \in K$. With each section $q=(u, v) \in \mathcal{T}_{k}, k \in K$, we associate a reduced graph $G^{q, k}=\left(V^{q, k}, E^{q, k}\right)$ in which a path joining the successive terminals $u$ to $v$ has to be found. The reduced graph $G^{q, k}$ is obtained from the original one by deleting all the terminals of $T_{k}$, except $u$ and $v$, as well as their incident edges.

Given $W \subseteq V$, we denote by $\delta_{G}(W)$ the set of edges of $E$ having exactly one vertex in $W$. The edge set $\delta_{G}(W)$ is called a cut. If $W \subset V, \bar{W}$ denotes $V \backslash W$. For $W=\{v\}$ for some $v \in V$, we will write $\delta(v)$ for $\delta(\{v\})$. Given two consecutive terminals of a section $q=(u, v) \in \mathcal{T}_{k}$ of a salesman $k \in K$, and a set of nodes $W \subseteq V^{q, k}$, with $u \in W$ and $v \in \bar{W}=V^{q, k} \backslash W$, the cut $\delta_{G^{q, k}}(W)$ in $G^{q, k}$ is called a section cut.

Figure 3 illustrates the notion of sections' reduced graphs. Figure 3 a presents a graph with an ordered set of terminals $\left(v_{1}, v_{4}, v_{3}\right)$. Consider section $q=\left(v_{4}, v_{1}\right)$. The corresponding reduced graph given in Fig. $3 \mathrm{~b}$ is obtained by deleting terminal $v_{3}$ as well as its incident edges. Here, the set $W=\left\{v_{1}, v_{2}, v_{5}, v_{7}\right\}$, for example, induces the section cut $\delta_{G^{q, k}}(W)=\left\{e_{2}, e_{6}, e_{9}\right\}$.

\subsection{Complexity}

The purpose of this section is to show that the MSTSPO is NP-hard. For this, we will show that even the restricted variant of the MSTSPO with a single tour, denoted by STSPO, is also NP-hard. We shall use a reduction from the $k$-vertex (a)

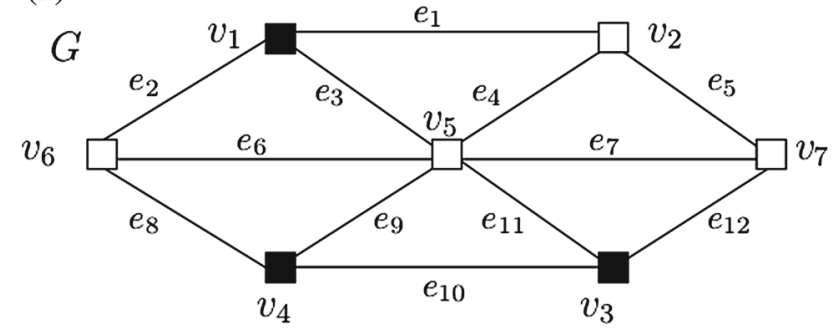

(b)

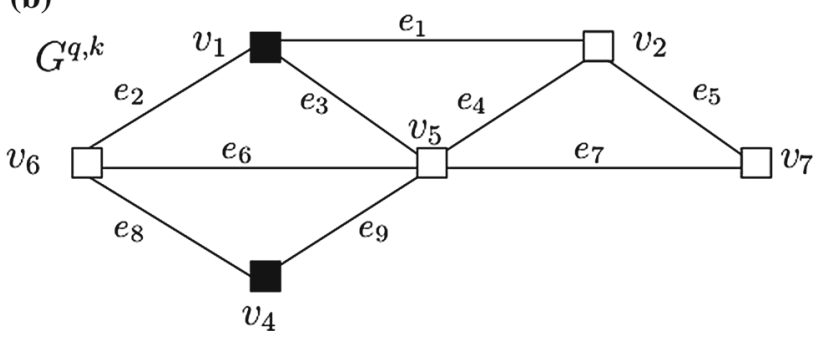

Fig. 3 Reduced graph for section $q=\left(v_{4}, v_{1}\right)$

disjoint paths problem. An instance of the $k$-vertex disjoint paths problem $(k \mathrm{VDPP})$ consists of a graph $\widetilde{G}=(\widetilde{V}, \widetilde{E})$, a weight vector $\widetilde{C}=\left(\tilde{c}_{e}, e \in \widetilde{E}\right) \geq 0$ associated with the edges of $\widetilde{E}$, a set of pairs of origin-destinations $\widetilde{T}=\left\{\left(s_{i}, t_{i}\right), i=1, \ldots, k\right\}, k \geq 3$ and a nonnegative scalar $M$. The problem consists in determining whether or not there exists $k$-node disjoint paths between the origin-destinations whose total weight does not exceed $M$. The $k \mathrm{VDPP}$ has been shown to be NP-hard Kobayashi and Sommer (2010). 


\section{Theorem 1 The STSPO is NP-hard.}

Proof It is clear that the STSPO problem is in NP. We will show that the $k$ VDPP reduces to the decision version of the STSPO: Given a graph $G=(V, E)$, a weight vector $C=$ $\left(c_{e}, e \in E\right) \geq 0$ associated with the edges of $E$, a set of terminals $T=\left\{v_{1}, \ldots, v_{l}\right\} \subseteq V$, and a positive scalar $M$, the problem consists in determining whether or not there exists in $G$ an elementary tour going in order through the terminals and whose weight does not exceed $M$. To this end, consider an instance $(\widetilde{G}, \widetilde{C}, \widetilde{T}, M)$ of the $k$ VDPP where $\widetilde{G}=(\widetilde{V}, \widetilde{E})$. We shall construct from $(\widetilde{G}, \widetilde{C}, \widetilde{T}, M)$ an instance $(G, C, T, M)$ of the STSPO as follows. To $\tilde{G}$, we add $k$ vertices $u_{1}, \ldots, u_{k}$ and $2 k$ edges $\left\{t_{i}, u_{i}\right\},\left\{u_{i}, s_{i+1}\right\}, i=1, \ldots, k\left(s_{1}=s_{k+1}\right)$. Let $G=(V, E)$ be the resulting graph and denote by $E_{u}$ the set of the added edges. Let $c_{e}=\tilde{c}_{e}$ if $e \in \widetilde{E}$ and $c_{e}=0$ if $e \in E_{u}$ be weights associated with the edges of $G$ (see Fig. 4). Finally, set $T=\left(s_{1}, t_{1}, u_{1}, s_{2}, \ldots, s_{j}, t_{j}, u_{j} \ldots, s_{k}, t_{k}, u_{k}\right)$ as the ordered set of terminals in $G$.

In the following, we show that there exist $k$ vertex disjoint paths between the pairs of $\widetilde{T}$ in $\widetilde{G}$ with total weight $\leq M$ if and only if there exists in $G$ an elementary tour going in order through the terminals of $T$ with total weight $\leq M$. Consider first a solution of the $k$ VDPP in $\widetilde{G}$ with a total weight $c \leq M$. The solution consists of $k$ vertex disjoint paths between the pairs $\left(s_{i}, t_{i}\right), i=1, \ldots, k$. These, together with the set of edges of $E_{u}$, constitute an elementary tour in $G$ going in order through the terminals of $T$. As the weights of the edges in $E_{u}$ are equal to 0 , the weight of the tour is equal to $c \leq M$. Consider now an elementary tour in $G$ going in order through the terminals $T$ with a total weight $c \leq M$ and consider the sections of the tour between the terminals $\left(s_{i}, t_{i}\right), i=$ $1, \ldots, k$. Clearly, these sections are node disjoint. Moreover, as the weights of all edges in $E_{u}$ are 0 , the total weight of the sub-paths between $\left(s_{i}, t_{i}\right), i=1, \ldots, k$ is exactly equal to $c$ which is $\leq M$.

Therefore, the STSPO is NP-hard, and so is the MSTSPO in consequence.

In the following sections, we propose two integer linear programming formulations and devise algorithms to solve the MSTSPO. We will assume in the sequel that $G$ is a complete graph.

\section{Integer programming formulations}

In this section, integer programming formulations are proposed for the MSTSPO. The first is a cut formulation.

\subsection{Cut formulation}

Let $x_{e}^{q, k}, e \in E, k \in K, q \in \mathcal{T}_{k}$ be a binary variable that takes value 1 if edge $e$ is used to route section $q$ of tour $k$ and 0 otherwise. These $x$ variables are called routing variables. Let $y_{e}, e \in E$ be a variable which takes value 1 if $e$ is installed and 0 otherwise. The $y$ variables are called design variables. The cut formulation for the MSTSPO is given by the following integer linear program,

$$
\begin{array}{ll}
\min \sum_{e \in E} c(e) y_{e} & \\
\sum_{e \in \delta_{G^{q, k}}(W)} x_{e}^{q, k} \geq 1 & \begin{array}{l}
\text { for all } k \in K, q=(u, v) \in V^{q, k}: u \in W \text { and } v \in \bar{W} \\
W
\end{array} \\
\sum_{q \in \mathcal{T}_{k}} \sum_{e \in \delta_{G}(v)} x_{e}^{q, k} \leq 2 & \text { for all } v \in V, k \in K \\
\sum_{q \in \mathcal{T}_{k}} x_{e}^{q, k} \leq y_{e} & \text { for all } e \in E, k \in K \\
x_{e}^{q, k} \in\{0,1\} & \text { for all } e \in E, k \in K, q \in \mathcal{T}_{k} \\
y_{e} \in\{0,1\} & \text { for all } e \in E .
\end{array}
$$

The above formulation will be called Cut Formulation and denoted by $(Q)$. Inequalities (1) are called section cut inequalities. They ensure that between the terminals of each section of tour $k \in K$, there is a path not going through any other terminal of $k$. Inequalities (2), called node-disjunction inequalities, ensure that these paths are node disjoint, and thus, they form an elementary tour. Inequalities (3) are the linking inequalities. These express the fact that an edge corresponding to a link which is not installed cannot be used to route any section. Constraints (4) and (5) are the integrality constraints.

There are an exponential number of inequalities (1). In order to separate them, for each $k \in K$ and each $q=(u, v) \in$ $\mathcal{T}_{k}$, a minimum $(u-v)$-cut in graph $G^{q, k}$ should be found. This naturally gives rise to a Branch-and-Cut algorithm over the Cut Formulation.

\subsection{Path formulation}

In this section, we propose a Dantzig-Wolfe decomposition of the integer linear program given by (1)-(5). Let $P^{q, k}$ be the set of paths that can route section $q \in \mathcal{T}_{k}$ of tour $k \in K$. Recall that these paths are computed in the reduced graph $G^{q, k}$. Let $z_{p}^{q, k}, k \in K, q \in \mathcal{T}_{k}, p \in P^{q, k}$ be a binary variable that takes 1 if path $p \in P^{q, k}$ is selected to route section $q$ of tour $k$ and 0 otherwise. The $z$ variables are called path variables and are linked to the routing variables by the following relation

$$
x_{e}^{q, k}=\sum_{p \in P^{q, k}} b_{p}(e) z_{p}^{q, k},
$$




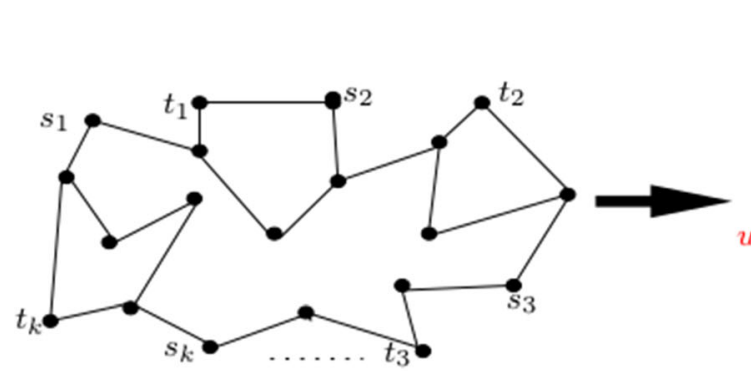

$\widetilde{G}$

Fig. 4 Reduction of the $k$ VDPP to the STSPO

where coefficients $b_{p}(e)$ takes 1 if edge $e \in p$ and 0 otherwise.

We also define coefficients $a_{p}(v)$ by

$a_{p}(v)=\left\{\begin{array}{l}1 \text { if vertex } v \text { is one of the extremities of path } p, \\ 2 \text { if } v \text { is an interior vertex of } p, \\ 0 \text { otherwise. }\end{array}\right.$

Using these notations, we have the following integer programming formulation for the MSTSPO

$$
\begin{aligned}
& \min \sum_{e \in E} c(e) y_{e} \\
& \sum_{p \in P^{q, k}} z_{p}^{q, k} \geq 1 \quad \text { for all } k \in K, q \in \mathcal{T}_{k}, \quad \pi^{q, k} \\
& \sum_{q \in \mathcal{T}_{k}} \sum_{p \in P^{q, k}} a_{p}(v) z_{p}^{q, k} \leq 2 \quad \text { for all } v \in V, k \in K, \quad \lambda_{v}^{k}
\end{aligned}
$$

$\sum_{q \in \mathcal{T}_{k}} \sum_{p \in P, k} b_{p}(e) z_{p}^{q, k} \leq y_{e} \quad$ for all $e \in E, k \in K, \quad \beta_{e}^{k}$

$$
\begin{array}{ll}
y_{e} \in\{0,1\} & \text { for all } e \in E \\
z_{p}^{q, k} \in\{0,1\} & \text { for all } k \in K, q \in \mathcal{T}_{k}, p \in P^{q, k} .
\end{array}
$$

The above formulation will be called Path Formulation and denoted by $(P)$. Note that $(P)$ contains an exponential number of $z$ variables. Let $\pi^{q, k}, \lambda_{w}^{k}$ and $\beta_{e}^{k}$ be the dual variables associated with inequalities (7), (8) and (9), respectively. These will be needed for the sequel.

The linear relaxations of the Cut and Path formulations yield exactly the same bound. This happens because the Path formulation can be viewed as arising from a Dantzig-Wolfe decomposition of the Cut formulation where Inequalities (1) and (4) define the subproblems. As those subproblems have the integrality property, the bounds are identical (Geoffrion

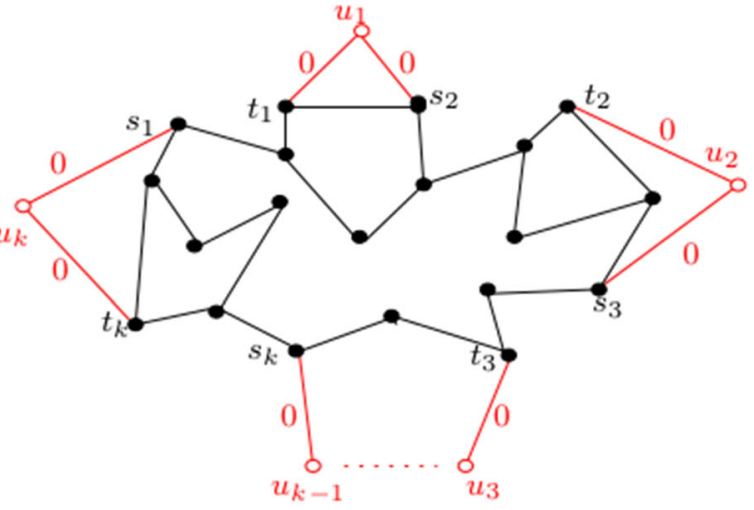

$G$
1974). However, it is far from clear which formulation will perform better in practice. A Branch-and-Cut over the Cut Formulation $(Q)$ requires separation of an exponential number of constraints using a minimum cut algorithm. On the other hand, a Branch-and-Price over the Path formulation $(P)$ requires pricing over an exponential number of variables using a shortest path algorithm. Branch-and-Cut algorithms are much more standard (for example, see Wolsey 1998) than Branch-and-Price algorithms. Therefore, in the following section, we only describe in detail the proposed Branch-andPrice algorithm over $(P)$.

\section{Branch-and-Price Algorithm}

As $(P)$ has a huge number of variables, solving its linear relaxation requires a column generation method. The combination of column generation with the Branch-and-Bound technique yields a Branch-and-Price algorithm for solving $(P)$. The idea of column generation is to solve a sequence of linear programs containing a restricted number of $z$ variables (called also columns), defining the Restricted Linear Programs (RLP). Starting from an initial linear program $\left(\mathrm{RLP}_{\text {ini }}\right)$ with a reasonable number of variables, at each iteration of the algorithm, we solve a satellite problem called the pricing problem. The pricing aims at detecting additional columns with negative reduced cost. If no such column exists, the current (RLP) is optimal. However, if its solution is fractional, a branching should be performed. Algorithm 1 summarizes the steps of our Branch-and-Price algorithm. For a deeper idea on the Branch-and-Price approach and its applications, the reader can refer to Desaulniers et al. (2005) and Lübbecke and Desrosiers (2005).

In the following sections, we describe in more details the main steps of Algorithm 1. 


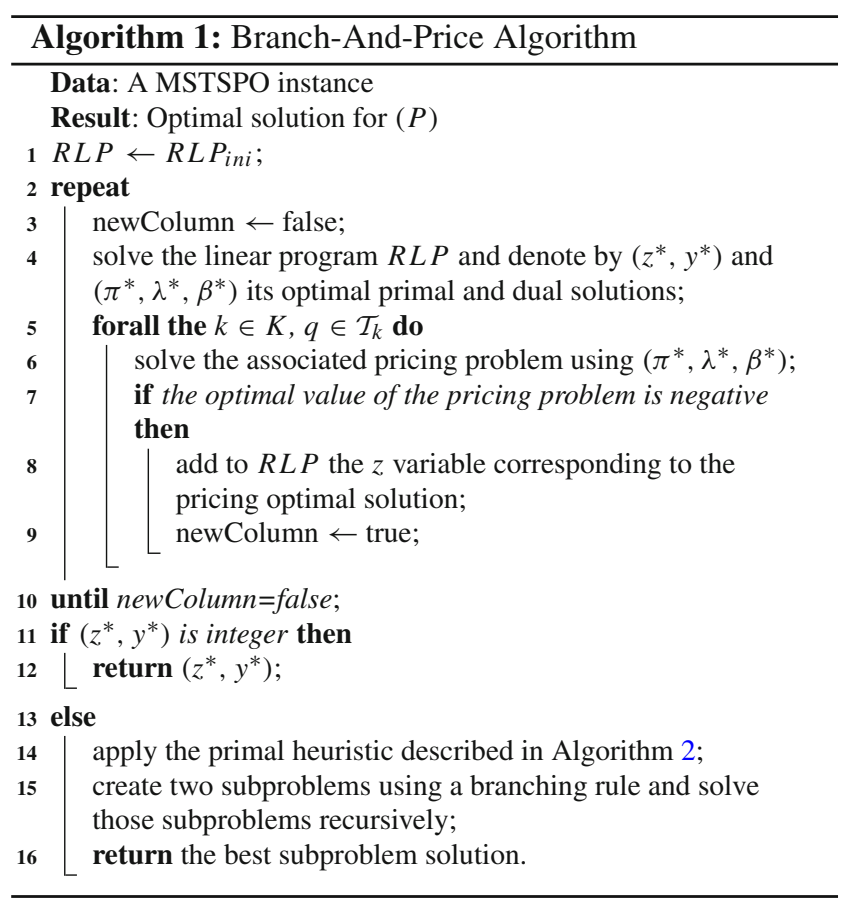

\subsection{Initial linear program RLPini}

For each section, $q=(u, v)$ of a tour $k \in K$, let $P_{0}^{q, k}$ be the subset of $P^{q, k}$ given by the paths between $u$ and $v$, of the form $(u, v),\left(u, s_{1}, v\right)$ and $\left(u, s_{1}, s_{2}, v\right)$, where $s_{1}, s_{2} \in S_{k}$ (recall that $S_{k}=V \backslash T_{k}$ is the set of Steiner nodes). (u,v) is called a direct-path for section $q$. We may assume that those initial paths always exist because, if needed, we may add edges with very large costs to $G$ without changing the optimal solution. Our first linear program $\mathrm{RLP}_{\text {ini }}$ is given by the columns corresponding to the paths of $\bigcup_{q, k} P_{0}^{q, k}$. Since the $\left|T_{k}\right|$ direct paths routing the sections of each tour $k$ form an elementary tour going in order through the terminals in $T_{k}$, it is clear that $\mathrm{RLP}_{\text {ini }}$ is feasible. The purpose of also having the paths with one or two Steiner vertices in RLP $_{\text {ini }}$ is for accelerating the Branch-and-Price, reducing the number of columns that have to be generated along the algorithm. Experiments have shown that, for most of the instances, it is not worthy to include paths with three or more Steiner vertices in the initialization, since they make the RLP solution too slow.

\subsection{Pricing problem}

The Branch-and-Price algorithm works over a restricted linear program involving only a subset of the $z$ variables. Additional columns will then be generated when they are needed. This can be achieved by solving the so-called pricing problem.
For every demand $k \in K$ and section $q \in \mathcal{T}_{k}$, we consider a pricing problem which consists in finding a new variable $z_{p}^{q, k}$ with negative reduced cost. The reduced cost for variable $z_{p}^{q, k}$ is given by

$$
R_{p}^{q, k}=-\left(\pi^{q, k}+\sum_{v \in V^{q, k}} a_{p}(v) \lambda_{v}^{k}+\sum_{e \in E^{q}, k} b_{p}(e) \beta_{e}^{k}\right) .
$$

Therefore, given an optimal dual solution $(\pi, \lambda, \beta)$ of RLP, the pricing problem consists in finding a path $p^{*}$ of $P^{q, k}$ such that

$$
R_{p^{*}}^{q, k}=\min _{p \in P_{k}^{q}} R_{p}^{q, k} . \quad(\operatorname{PS}(q, k))
$$

If $R_{p^{*}}^{q, k}<0$, then the column related to variable $z_{p^{*}}^{q, k}$ is added to RLP. Otherwise, $R_{p^{*}}^{q, k} \geq 0$ for all $k \in K$ and $q \in \mathcal{T}_{k}$, which means that the optimal solution of the current linear program (RLP) is optimal for the linear relaxation of the path formulation $(P)$.

In what follows, we will show that the pricing problem $\operatorname{PS}(q, k)$ reduces to a shortest path problem in the reduced graph $G^{q, k}$. Suppose $q=\left(v_{j}, v_{j+1}\right)$. Associate with each edge $e=u v$ of graph $G^{q, k}$, the length $l_{e}$ is given by

$l_{e}=-\beta_{e}^{k}-\lambda_{u}^{k}-\lambda_{v}^{k}$

See Fig. 5 for an illustration that considers the reduced graph of Fig. 3 corresponding to section $q=\left(v_{4}, v_{1}\right)$.

Lemma 2 Given a path $p \in P^{q, k}, R_{p}^{q, k}=-\pi^{q, k}+l(p)$, where $l(p)$ is the length of $p$.

Proof Assume $p$ is the sequence of vertices and edges $\left(v_{j}, e_{1}, u_{1}, \ldots, u_{t-1}, e_{t}, v_{j+1}\right)$. Thus,

$$
\begin{aligned}
R_{p}^{q, k} & =-\pi^{q, k}-\sum_{v \in V^{q, k}} a_{p}^{q, k}(v) \lambda_{v}^{k}-\sum_{e \in E^{q, k}} b_{p}^{q, k}(e) \beta_{e}^{k} \\
& =-\pi^{q, k}+l(p) .
\end{aligned}
$$

By Lemma 2, minimizing $R_{p}^{q, k}$ reduces to minimizing $l(p)$, that is to say solving a shortest path problem in the reduced graph $G^{q, k}$. Note that the dual variables $\lambda$ and $\beta$ are non-positive. Hence, edge lengths are nonnegative and the shortest path problem can be solved in polynomial time using, for instance, Dijkstra Algorithm (Dijkstra 1959).

\subsection{Branching scheme}

At each node of the Branch-and-Price tree, if the optimal solution $\left(z^{*}, y^{*}\right)$ of the associated RLP is not integer, a 
Fig. 5 Reduced graph with dual weights on edges

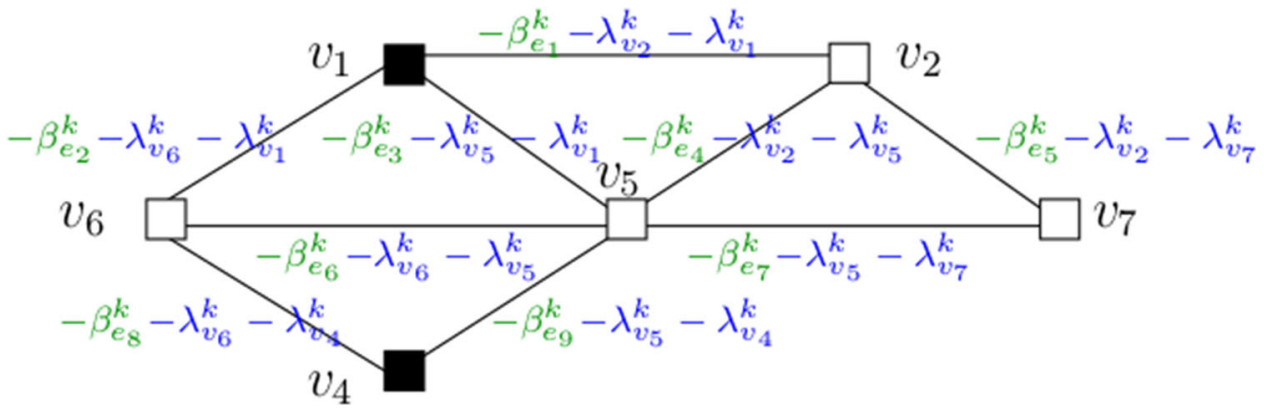

branching must be performed. If possible, this is done over the design variables. If there are $y$ variables with fractional value, the most fractional such variable is selected, say $y_{e}^{*}$. On the first subproblem, $y_{e}^{*}$ is fixed to 0 , on the second $y_{e}^{*}$ is fixed to 1 . Notice that this kind of branching does not change the pricing since it does not fix specific path variables.

However, at a certain level of the tree, we may have a fractional solution where all the design variables are integral. In this case, a branching on the path variables is necessary. The challenge is to identify a branching rule that eliminates the current fractional solution without compromising the tractability of the pricing problem. In general, the branching rules for path-based formulations are defined on the original edges (or arcs) of the paths (Barnhart et al. 2000; Feillet 2010). For example, we can branch as follows: for a given $k \in K$ and $q \in \mathcal{T}_{k}$, on the first subproblem we forbid the use of a certain edge $e$ on the path that connects that section; on the second, we impose that it passes by $e$. This amounts to branching on the following constraints:

- $\sum_{p \in P^{q, k}} b_{p}^{q, k}(e) z_{p}^{q, k} \leq 0$ for the first subproblem, - and $\sum_{p \in P^{q, k}} b_{p}^{q, k}(e) z_{p}^{q, k} \geq 1$ for the second subproblem.

The dual variables of these constraints change the reduced cost calculation when solving $\operatorname{PS}(q, k)$. For the first constraint, this poses no problem, since the new $l_{e}$ will still be nonnegative. However, the dual variable of the second constraint may be positive, resulting in a negative edge length $l_{e}$. This may yield negative cycles in the graph. Hence, the shortest path problems in the pricing can no longer be solved with a polynomial algorithm.

This problem can be avoided by the following observation. Path Formulation (7)-(11) remains correct if, for each section $q=(u, v)$ of a tour $k$, the set $P^{q, k}$ is replaced by a larger set $\bar{P}^{q, k}$ containing all subsets of edges in $G^{q, k}$ that contain a path joining $u$ to $v$. In other words, the elements $p \in \bar{P}^{q, k}$ are formed by a $u-v$-path plus, possibly, other edges. The definition of $\bar{P}^{q, k}$ is not arbitrary, and it would appear as a result of a direct Dantzig-Wolfe decomposition of the Cut formulation. In fact, the elements of $\bar{P}^{q, k}$ correspond exactly to the binary points that satisfy the corresponding constraints in (1). However, since there is always an optimal solution without variables $z_{p}^{q, k}$ such that $p \in\left(\bar{P}^{q, k} \backslash P^{q, k}\right)$, we opted for the simpler definition. Anyway, if all reduced costs $l_{e}$ are nonnegative, then $\min _{p \in P_{k}^{q}} R_{p}^{q, k}=\min _{p \in \bar{P}_{k}^{q}} R_{p}^{q, k}$, so the two definitions are equivalent.

When the branching constraints make some reduced costs $l_{e}$ negative, we perform the pricing over $\bar{P}^{q, k}$. This is done as follows:

1. Let $N$ be the set of edges with negative $l_{e}$ and include all those edges in the optimal solution $p^{*}$.

2. Take $G^{q, k}$ and contract all edges in $N$. The resulting graph does not have negative edges anymore.

3. Use Dijkstra's algorithm for finding the shortest path between $u$ and $v$ in the contracted graph and add this path to $p^{*}$.

In this way, the complexity of the pricing never changes.

\subsection{Primal heuristic}

In order to accelerate the Branch-and-Price algorithm, we propose a primal heuristic that enables the improvement in the upper bound and a faster pruning of the Branch-andBound tree. Given a fractional solution, a feasible integer solution for the MSTSPO can be determined using the primal heuristic described in Algorithm 2. This is a greedy-like algorithm. We start from a feasible fractional solution $(z, y)$. For each demand $k \in K$, we go through the different sections from $\left(v_{1}, v_{2}\right)$ to $\left(v_{\left|T_{k}\right|}, v_{\left|T_{k}\right|+1}\right)$ with $v_{\left|T_{k}\right|+1}=v_{1}$. At step $i$ of Algorithm 2, our objective is to select a path for routing section $q=\left(v_{i}, v_{i+1}\right)$ among the paths corresponding to the nonzero variables $z_{p}^{q, k}$. This path must respect the disjunction constraints with paths already chosen for routing the previous sections.

In Algorithm 2, $S_{k,+}$ represents the Steiner nodes that have been used to route the $(i-1)$ first sections of tour $k$. Considering section $q=\left(v_{i}, v_{i+1}\right)$, we first begin by sorting in non-increasing order the variables $z_{p}^{q, k}$ by their values. Let $z_{p_{1}}^{q, k} \geq z_{p_{2}}^{q, k} \geq \cdots \geq z_{p_{h}}^{q, k}$ be the sorted variables represent- 
ing, respectively, paths $p_{1}, p_{2}, \ldots, p_{h}$ routing $q \in \mathcal{T}_{k}$. The first path $p_{j}$ such that its set of Steiner nodes $S p_{j}$ is disjoint from $S_{k,+}$ is selected $\left(\hat{z}_{p_{j}}^{q, k}=1\right)$ and $S p_{j}$ is added to $S_{k,+}$. If none of the paths $p_{1}, p_{2}, \ldots, p_{h}$ has been selected; then, the section is routed by the path $p_{0}=\left(v_{i}, v_{i+1}\right)\left(\hat{z}_{p_{0}}^{q, k}=1\right)$. Once a path is selected to route section $q=\left(v_{i}, v_{i+1}\right)$, the next section is considered and the same procedure is applied.

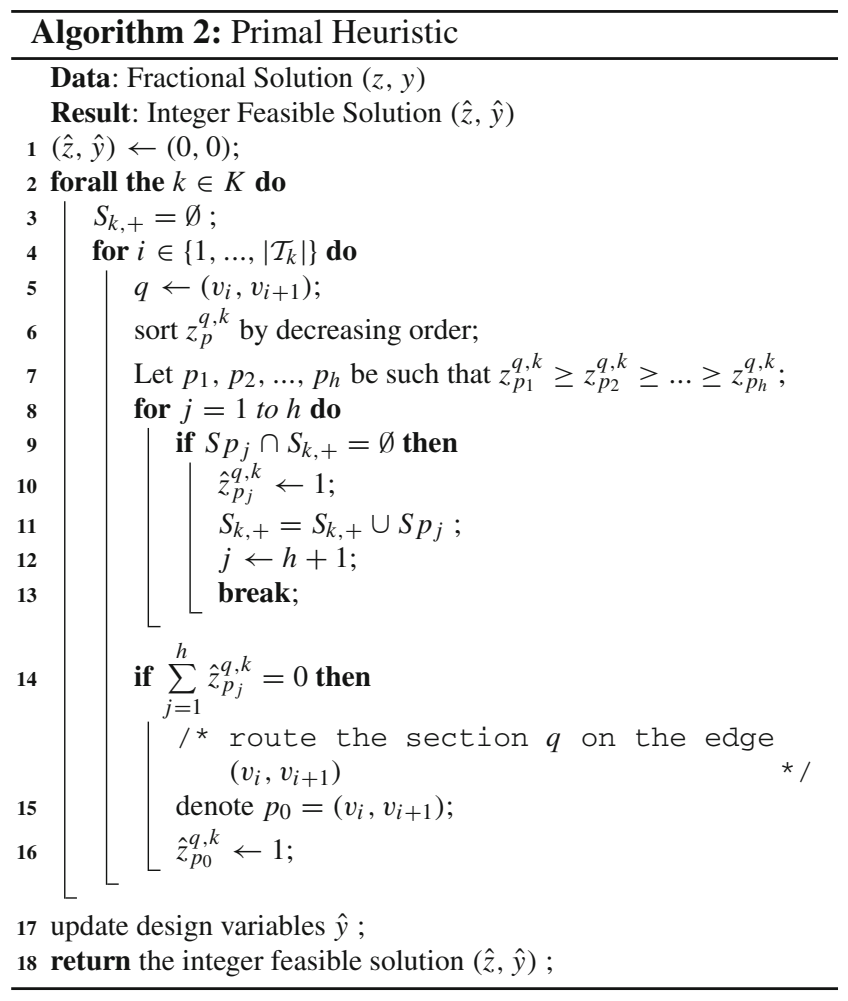

The primal heuristic described in Algorithm 2 is applied at the end of the column generation phase for each node of the Branch-and-Price tree.

\section{Computational results}

The Branch-and-Cut and Branch-and-Price algorithms described in the previous sections are implemented in $\mathrm{C}++$, using ABACUS 3.2 (http://www.informatik.uni-koeln.de/ abacus/) to manage the Branch-and-Cut and Branch-andPrice trees and Cplex 12.8 as LP-solver (http://www.ilog. $\mathrm{com} /$ products/cplex/). The tests are performed on a Bi-Xeon quad-core E5507 2.27GHz machine with $8 \mathrm{~Gb}$ of RAM, running under Linux. The maximum CPU time is fixed to $3 \mathrm{~h}$.

Two types of instances were tested: random instances generated from the TSP-Library (http://comopt.ifi.uni-heidelberg. de/software/TSPLIB95/) instances (a280, bier 127, eil101, lin 105 and tsp225) and realistic instances generated from the SNDLib (http://sndlib.zib.de/) instances ( $d f n-b w i n$, polska, nobel - us, newyork and geant). All the instances have complete graphs and costs corresponding to Euclidian distances.

In the sequel, the entries of the different tables are :

\begin{tabular}{|c|c|}
\hline Inst: & $\begin{array}{l}\text { Name of the original instance (for } \\
\text { SNDLib instances) }\end{array}$ \\
\hline$|V|:$ & Number of vertices in graph $G$ \\
\hline$|K|:$ & Number of salesmen \\
\hline$|T|:$ & Average number of terminals \\
\hline NC (resp. NP): & $\begin{array}{l}\text { Number of nodes in the Branch- } \\
\text { and-Cut tree (resp. Branch-and- } \\
\text { Price tree) }\end{array}$ \\
\hline Path-ini: & $\begin{array}{l}\text { Number of columns provided by the } \\
\text { initial solution }\end{array}$ \\
\hline Path-gen: & $\begin{array}{l}\text { Number of columns generated dur- } \\
\text { ing the pricing }\end{array}$ \\
\hline Gap-C (resp. Gap-P): & $\begin{array}{l}\text { The relative error between the best } \\
\text { upper bound (the optimal solution } \\
\text { if the problem has been solved to } \\
\text { optimality) and the lower bound } \\
\text { obtained at the root node of the } \\
\text { Branch-and-Cut tree (resp. Branch- } \\
\text { and-Price tree) }\end{array}$ \\
\hline Opt-C (resp. Opt-P): & $\begin{array}{l}\text { Number of instances in the group } \\
\text { solved to optimality by the Branch- } \\
\text { and-Cut (resp. Branch-and-Price) } \\
\text { algorithm (for the random instances } \\
\text { only) }\end{array}$ \\
\hline Time-C (resp. Time-P): & $\begin{array}{l}\text { Total CPU time in seconds for the } \\
\text { Branch-and-Cut (resp. Branch-and- } \\
\text { Price) algorithm }\end{array}$ \\
\hline
\end{tabular}

The reported results for the random set of instances are averages over the five tested instances mentioned above. In addition, when it is not possible to find a feasible solution within the time limit, a "-" appears in the Gap column (for the Branch-and-Cut algorithm).

Table 1 reports the results for the random instances for both the Branch-and-Cut (B\&C) and Branch-and-Price (B\&P) algorithms. It can be seen that neither algorithm dominates the other. Both are efficient for small instances, for which optimality is reached for all the 5 instances in a group. For medium-sized instances, the $\mathrm{B} \& \mathrm{C}$ algorithm seems to perform better than the $\mathrm{B} \& \mathrm{P}$ algorithm for smaller values of $|K|$, say, up to 10. In fact, all instances in some groups could be solved to optimality within some seconds by the B\&C, and none of those instances could be solved within the time limit using the B\&P. This happens in the group with $|V|=16$ and $|K|=5$ and in the group with $|V|=20$ and $|K|=5$. On the other hand, the $\mathrm{B} \& \mathrm{P}$ performs better than the $\mathrm{B} \& \mathrm{C}$ on groups like $|V|=12$ and $|K|=20$. In that group, all the five instances could be solved with the $\mathrm{B} \& \mathrm{P}$ algorithm, with an average time of $22 \mathrm{~min}$, while the $\mathrm{B} \& \mathrm{C}$ could only solve one instance within the time limit. For the larger instances, 
Table 1 Branch-and-Cut versus Branch-and-Price results for random instances

\begin{tabular}{|c|c|c|c|c|c|c|c|c|c|c|c|c|c|}
\hline$|V|$ & $|K|$ & $|T|$ & $\mathrm{NC}$ & Cuts & Gap-C & Opt-C & Time-C & NP & Path-ini & Path-gen & Gap-P & Opt-P & Time-P \\
\hline 8 & 2 & 4.50 & 1 & 80.4 & 0.00 & $5 / 5$ & 0.36 & 1 & 118 & 0 & 0.00 & $5 / 5$ & 0.27 \\
\hline 8 & 5 & 4.20 & 11 & 226.2 & 3.62 & $5 / 5$ & 0.99 & 8.6 & 314 & 4.8 & 3.62 & $5 / 5$ & 1.12 \\
\hline 8 & 10 & 4.10 & 13 & 473.2 & 2.19 & $5 / 5$ & 1.14 & 8.2 & 646 & 2.6 & 2.19 & $5 / 5$ & 0.96 \\
\hline 8 & 15 & 4.07 & 19.8 & 729 & 3.18 & $5 / 5$ & 3.60 & 13 & 986 & 3 & 3.18 & $5 / 5$ & 1.27 \\
\hline 12 & 2 & 6.50 & 25 & 206 & 2.87 & $5 / 5$ & 1.43 & 15.8 & 404 & 16.8 & 2.87 & $5 / 5$ & 2.65 \\
\hline 12 & 5 & 5.80 & 121.8 & 795.6 & 5.46 & $5 / 5$ & 19.39 & 119.8 & 1096 & 331.6 & 5.46 & $5 / 5$ & 473.69 \\
\hline 12 & 10 & 5.70 & 146.6 & 2099.8 & 7.12 & $5 / 5$ & 89.93 & 123.8 & 2232 & 293.8 & 7.12 & $5 / 5$ & 90.54 \\
\hline 12 & 15 & 6.00 & 1358.6 & 4263 & 9.44 & $5 / 5$ & 1180.15 & 1117 & 3204 & 530.4 & 9.44 & $5 / 5$ & 813.58 \\
\hline 12 & 20 & 6.10 & 4115.8 & 5856.2 & 10.47 & $1 / 5$ & 8837.52 & 1023.4 & 4216 & 429.8 & 9.36 & $5 / 5$ & 1321.80 \\
\hline 16 & 2 & 4.50 & 1 & 268.8 & 0.00 & $5 / 5$ & 3.25 & 1 & 1190 & 80 & 0.00 & $5 / 5$ & 1.81 \\
\hline 16 & 5 & 4.80 & 642.2 & 3298 & 5.46 & $5 / 5$ & 309.85 & 14 & 2912 & 1389.2 & 59.04 & $0 / 5$ & 10800 \\
\hline 16 & 10 & 5.40 & 1465.6 & 15370 & 6.63 & $3 / 5$ & 6234.81 & 403.4 & 5802 & 6711 & 64.04 & $0 / 5$ & 10800 \\
\hline 16 & 15 & 5.93 & 849.6 & 21268.2 & - & $0 / 5$ & 10800 & 847 & 8628 & 6364.4 & 83.70 & $0 / 5$ & 10800 \\
\hline 16 & 20 & 5.80 & 477.8 & 20810.8 & - & $0 / 5$ & 10800 & 684.4 & 11484 & 4566.2 & 86.92 & $0 / 5$ & 10800 \\
\hline 20 & 2 & 4.50 & 1 & 392.6 & 0.00 & $5 / 5$ & 1.11 & 1 & 2158 & 165.2 & 0.00 & $5 / 5$ & 2.90 \\
\hline 20 & 5 & 4.60 & 2651.8 & 7314.2 & 4.29 & $5 / 5$ & 3456.10 & 1 & 5340 & 2026.4 & 59.48 & $0 / 5$ & 10800 \\
\hline 20 & 10 & 5.60 & 397 & 28001 & - & $0 / 5$ & 10800 & 110 & 11060 & 12182.2 & 77.26 & $0 / 5$ & 10800 \\
\hline 20 & 15 & 5.67 & 93.4 & 21328.2 & - & $0 / 5$ & 10800 & 47.2 & 16874 & 9315.6 & 84.28 & $0 / 5$ & 10800 \\
\hline 20 & 20 & 5.45 & 38 & 18117.2 & - & $0 / 5$ & 10800 & 28.4 & 22222 & 7326.2 & 87.70 & $0 / 5$ & 10800 \\
\hline 25 & 2 & 4.50 & 1 & 553.4 & 0.00 & $5 / 5$ & 5.15 & 1 & 3627 & 260.6 & 0.00 & $5 / 5$ & 14.46 \\
\hline 25 & 5 & 5.40 & 904.6 & 20256.2 & - & $0 / 5$ & 10800 & 1 & 9677 & 3076.6 & 58.40 & $0 / 5$ & 10800 \\
\hline 25 & 10 & 5.40 & 38.2 & 15041.6 & - & $0 / 5$ & 10800 & 2.8 & 19902 & 7470.6 & 74.48 & $0 / 5$ & 10800 \\
\hline 25 & 15 & 5.27 & 6 & 12995.8 & - & $0 / 5$ & 10800 & 1.6 & 29711 & 8455.6 & 82.35 & $0 / 5$ & 10800 \\
\hline 25 & 20 & 5.50 & 1 & 10652.2 & - & $0 / 5$ & 10800 & 1.8 & 40208 & 6835.6 & 86.53 & $0 / 5$ & 10800 \\
\hline
\end{tabular}

mainly for $|V| \geq 20$, and $|K| \geq 10$, none of the algorithms could obtain optimal solutions. However, the B\&P algorithm can at least provide feasible solutions (some with large gaps exceeding 70\%).

Overall, among the 24 groups of instances, 13 were completely solved to optimality by the $\mathrm{B} \& \mathrm{C}$ algorithm and 12 by the $\mathrm{B} \& \mathrm{P}$ algorithm. In addition, for those groups where all optimal solution values are known, root gaps do not exceed $10 \%$ even for instances with 15 and 20 salesmen, which indicates that the quality of the linear relaxations of the proposed formulations is good. However, there is space for future investigation of additional valid inequalities that may reduce those gaps.

In order to see our algorithms dealing with more realistic instances, we tested them on SNDLib instances. The results are reported in Table 2 for 30 instances with a number of vertices ranging from 11 to 23 and a number of salesmen going from 5 to 40 . It can be seen that both algorithms could solve 20 instances within the time limit. The $\mathrm{B} \& \mathrm{C}$ does not perform well on instances with large $|K|$. In fact, it failed on all instances with $|K|=40$. On the other hand, it clearly performs better on instances with up to 15 salesmen. The average root gap over all the 20 instances solved to optimality was $2.46 \%$, and the maximum gap did not exceed $8 \%$.
Generally, the algorithms performed better on the realistic instances. This behavior is somehow expected. The random instances may have some demands where the terminals are very far apart, making the resolution harder.

\section{Concluding remarks}

In this paper, we have considered a variant of the TSP that is the Multiple Steiner TSP with Order constraints. We have shown that it is NP-hard even for one salesman. We have proposed integer programming formulations giving rise to Branch-and-Cut and Branch-and-Price algorithms. For the Branch-and-Price algorithm, we have shown that the pricing problem reduces to a shortest path problem and can then be solved in polynomial time. We have also proposed a branching strategy which does not change the pricing tractability. Moreover, we have devised a primal heuristic in order to accelerate the algorithm. Our computational results have shown that the algorithms perform reasonably well for medium-sized instances and that they are somehow complementary. Branch-and-Cut performed better on realistic instances with less salesmen, while Branch-and-Price is superior on realistic instances with many salesmen. 
Table 2 Branch-and-Cut versus Branch-and-Price results for realistic instances

\begin{tabular}{|c|c|c|c|c|c|c|c|c|c|c|c|c|}
\hline Instance & $|V|$ & $|K|$ & $|T|$ & $\mathrm{NC}$ & Cuts & Gap-C & Time-C & $\mathrm{NP}$ & Path-ini & Path-gen & Gap-P & Time-P \\
\hline dfn-bwin & 11 & 5 & 3.20 & 1 & 248 & 0.00 & 0.25 & 1 & 748 & 1 & 0.00 & 0.33 \\
\hline dfn-bwin & 11 & 10 & 3.40 & 1 & 551 & 0.00 & 0.55 & 1 & 1476 & 17 & 0.00 & 0.7 \\
\hline dfn-bwin & 11 & 15 & 3.33 & 1 & 819 & 0.00 & 0.81 & 1 & 2224 & 258 & 0.00 & 11 \\
\hline dfn-bwin & 11 & 20 & 3.45 & 2219 & 2628 & 8.00 & 1151.69 & 129 & 2966 & 1779 & 8.00 & 289.66 \\
\hline dfn-bwin & 11 & 30 & 3.50 & 9619 & 3778 & 12.50 & 10800 & 11 & 4454 & 674 & 2.00 & 82.49 \\
\hline dfn-bwin & 11 & 40 & 3.53 & 4668 & 6248 & - & 10800 & 43 & 5926 & 1038 & 5.17 & 266.62 \\
\hline polska & 13 & 5 & 3.80 & 15 & 453 & 2.50 & 3.47 & 15 & 1262 & 1045 & 2.50 & 44.15 \\
\hline polska & 13 & 10 & 3.90 & 67 & 1286 & 4.17 & 26.24 & 35 & 2472 & 1274 & 4.17 & 101.23 \\
\hline polska & 13 & 15 & 3.67 & 37 & 1932 & 5.45 & 40.83 & 45 & 3716 & 2681 & 5.45 & 420.66 \\
\hline polska & 13 & 20 & 3.65 & 21 & 2424 & 2.91 & 28.98 & 13 & 4988 & 2064 & 2.91 & 329.39 \\
\hline polska & 13 & 30 & 3.53 & 4528 & 5016 & 2.97 & 10800 & 15 & 7490 & 4620 & 2.97 & 1305.53 \\
\hline polska & 13 & 40 & 3.53 & 2622 & 4967 & - & 10800 & 39 & 10020 & 5583 & 4.67 & 3020.5 \\
\hline nobel-us & 15 & 5 & 3.80 & 3 & 469 & 0.00 & 2.3 & 3 & 1950 & 227 & 0.00 & 9.57 \\
\hline nobel-us & 15 & 10 & 3.40 & 1 & 859 & 0.00 & 1.56 & 1 & 3780 & 560 & 0.00 & 46.9 \\
\hline nobel-us & 15 & 15 & 3.60 & 39 & 1465 & 4.71 & 37.57 & 9 & 5710 & 798 & 4.71 & 126.57 \\
\hline nobel-us & 15 & 20 & 3.75 & 21 & 2486 & 1.72 & 43.88 & 9 & 7736 & 2833 & 1.72 & 935.42 \\
\hline nobel-us & 15 & 30 & 3.70 & 2159 & 8155 & - & 10800 & 34 & 11624 & 13585 & 3.55 & 10800 \\
\hline nobel-us & 15 & 40 & 3.63 & 1215 & 7782 & - & 10800 & 2 & 15436 & 6918 & 90.70 & 10800 \\
\hline newyork & 17 & 5 & 3.00 & 1 & 425 & 0.00 & 0.63 & 1 & 2550 & 126 & 0.00 & 7.99 \\
\hline newyork & 17 & 10 & 3.20 & 835 & 3929 & 7.29 & 886.48 & 4 & 5240 & 5572 & 9.56 & 10800 \\
\hline newyork & 17 & 15 & 3.40 & 181 & 3880 & 5.02 & 359.45 & 19 & 8030 & 12032 & 5.02 & 10800 \\
\hline newyork & 17 & 20 & 3.50 & 479 & 4172 & 4.05 & 1481.36 & 31 & 10820 & 11048 & 4.05 & 7997.38 \\
\hline newyork & 17 & 30 & 3.63 & 1041 & 9159 & - & 10800 & 23 & 16436 & 9686 & 3.28 & 8881.5 \\
\hline newyork & 17 & 40 & 3.60 & 619 & 9481 & - & 10800 & 2 & 21886 & 5383 & 3.16 & 10800 \\
\hline geant & 23 & 5 & 3.60 & 1 & 778 & 0.00 & 2.59 & 1 & 6008 & 2206 & 0.00 & 1.89 \\
\hline geant & 23 & 10 & 3.40 & 7 & 1977 & 0.00 & 79.88 & 1 & 11652 & 7869 & 43.89 & 10800 \\
\hline geant & 23 & 15 & 3.47 & 13 & 3431 & 0.24 & 203.79 & 1 & 17724 & 12106 & 55.28 & 10800 \\
\hline geant & 23 & 20 & 3.50 & 19 & 4901 & 0.28 & 602.71 & 1 & 23732 & 14071 & 68.46 & 10800 \\
\hline geant & 23 & 30 & 3.53 & 4 & 9629 & - & 10800 & 1 & 35748 & 41733 & 80.94 & 10800 \\
\hline geant & 23 & 40 & 3.55 & 1 & 8909 & - & 10800 & 1 & 47828 & 34805 & 83.44 & 10800 \\
\hline
\end{tabular}

There are many interesting extensions that could be considered in future works. It would, for example, be interesting to improve the linear relaxations of the algorithms by adding valid inequalities. This can be done within a deep investigation of the polyhedral structure of the problem. A further interesting extension would also be to add hop constraints on the tours

Acknowledgements The authors would like to thank Eric Gourdin and Nancy Perrot from Orange Labs, France, and Sylvie Borne from LIPN Univesité Paris 13, France, for their fruitful collaboration.

\section{Compliance with ethical standards}

Conflict of interest All authors declare that they have no conflict of interest.
Human and animal rights This article does not contain any studies with human participants or animals performed by any of the authors.

\section{References}

Applegate DL, Bixby RE, Chvatal V, Cook WJ (2011) The traveling salesman problem: a computational study. Princeton University Press, Princeton

Ascheuer N, Jünger M, Reinelt G (2000) A branch and cut algorithm for the asymmetric traveling salesman problem with precedence constraints. Comput Optim Appl 17:61-84

Baïou M, Mahjoub AR (2002) Steiner 2-edge connected subgraph polytopes on series-parallel graphs. SIAM J Discrete Math 10:505-514

Balas E, Fischetti M, Pulleyblank WR (1995) The precedenceconstrained asymmetric traveling salesman polytope. Math Program 68:241-265

Barnhart C, Hane CA, Vance PH (2000) Using branch-and-priceand-cut to solve origin-destination integer multicommodity Flow problem. Oper Res 48:318-326 
Bektas T (2006) The multiple traveling salesman problem: an overview of formulations and solution procedures. Omega 34(3):209-219

Benavent E, Martínez A (2013) Multi-depot multiple TSP: a polyhedral study and computational results. Ann Oper Res 207(1):7-25

Borne S, Virginie G, Mahjoub AR, Taktak R (2011) Multilayer survivable optical network design. In: International conference on network optimization. Springer, pp. 170-175

Borne S, Mahjoub AR, Taktak R (2013) A branch-and-cut algorithm for the multiple steiner TSP with order constraints. Electron Notes Discrete Math 41:487-494

Cornuéjols G, Fonlupt J, Naddef D (1985) The traveling salesman problem on a graph and some related integer polyhedra. Math Program 33:1-27

Dantzig G, Fulkerson R, Johnson S (1954) Solution of a large-scale traveling-salesman problem. J Oper Res Soc Am 2(4):393-410

Desaulniers G, Desrosiers J, Solomon MM (2005) Column generation, vol 5. Springer, Berlin

Dijkstra EW (1959) A note on two problems in connection with graphs. Numer Math 1:269-271

Feillet D (2010) A tutorial on column generation and branch-and-price for vehicle routing problems. J Oper Res 8:407-424

Geoffrion AM (1974) Lagrangean relaxation for integer programming. In: Approaches to integer programming. Springer, pp 82-114

Gouveia L, Pesneau P (2006) On extended formulations for the precedence constrained asymmetric traveling salesman problem. Networks 48(2):77-89

Gouveia L, Voß S (1995) A classification of formulations for the (timedependent) traveling salesman problem. Eur J Oper Res 83(1):6982

Gouveia L, Pesneau P, Ruthmair M, Santos D (2018) Combining and projecting flow models for the (precedence constrained) asymmetric traveling salesman problem. Networks 71(4):451-465

Grötschel M, Padberg MW (1979a) On the symmetric travelling salesman problem I: inequalities. Math Program 16(1):265-280

Grötschel M, Padberg MW (1979b) On the symmetric travelling salesman problem II: lifting theorems and facets. Math Program 16(1):281-302

Gutin G, Punnen AP (2006) The traveling salesman problem and its variations, vol 12. Springer, Berlin

Interian R, Ribeiro CC (2017) A grasp heuristic using path-relinking and restarts for the steiner traveling salesman problem. Int Trans Oper Res 24(6):1307-1323
Kobayashi Y, Sommer C (2010) On shortest disjoint paths in planar graphs. Discrete Optim 7(4):234-245

Letchford AN, Nasiri SD, Theis DO (2013) Compact formulations of the steiner traveling salesman problem and related problems. Eur J Oper Res 228(1):83-92

Lübbecke ME, Desrosiers J (2005) Selected topics in column generation. Oper Res 53(6):1007-1023

Mahjoub AR, Taktak R, Uchoa E (2019) A layered compact formulation for the multiple steiner TSP with order constraints. In: 2019 6th international conference on control, decision and information technologies (CoDIT). IEEE, pp 1462-1467

Moon C, Kim J, Choi G, Seo Y (2002) An efficient genetic algorithm for the traveling salesman problem with precedence constraints. Eur J Oper Res 140(3):606-617

Naddef D (2007) Polyhedral theory and branch-and-cut algorithms for the symmetric TSP. In: Gutin G, Punnen AP (eds) The traveling salesman problem and its variations. Springer, Boston, pp 29-116

Öncan T, Altınel İK, Laporte G (2009) A comparative analysis of several asymmetric traveling salesman problem formulations. Comput Oper Res 36(3):637-654

Orman AJ, Williams HP (2006) A survey of different integer programming formulations of the travelling salesman problem. Optim Econom Financ Anal 9:93-108

Padberg MW, Rinaldi G (1991) A branch-and-cut algorithm for the resolution of large-scale symmetric traveling salesman problems. SIAM Rev 33(1):60-100

Salazar-González JJ (2003) The steiner cycle polytope. Eur J Oper Res 147:671-679

Sarin SC, Sherali HD, Judd JD, Tsai P-FJ (2014) Multiple asymmetric traveling salesmen problem with and without precedence constraints: performance comparison of alternative formulations. Comput Oper Res 51:64-89

Steinová M (2012) Approximability of the minimum steiner cycle problem. Comput Inform 29(6):1349-1357

Wolsey LA (1998) Integer programming. Wiley, New York

Publisher's Note Springer Nature remains neutral with regard to jurisdictional claims in published maps and institutional affiliations. 